\title{
生物质谱在修饰酶底物篮选及动力学常数 测定中的应用
}

\author{
邓真真 1,2 , 王䶮 1,2 , 叶明亮 $1^{*}$ \\ 1. 中国科学院大连化学物理研究所, 中国科学院分离分析化学重点实验室, 国家色谱研究分析中心, 大连 116023; \\ 2. 中国科学院大学化学科学学院, 北京 100049 \\ *联系人, E-mail: mingliang@dicp.ac.cn \\ 收稿日期: 2017-08-01; 接受日期: 2017-09-06; 网络版发表日期: 2018-01-03 \\ 国家重点研发计划(批准号: 2016YFA0501402)和国家自然科学基金(批准号: 21525524)资助
}

摘要揭示酶与底物之间的对应关系是理解修饰酶相关信号调控网络的重要基础. 生物质谱可以实现对大
量丰度不同的底物进行定性及定量监测, 在复杂体系的酶促动力学的研究中具有重要地位. 本文简要综述了生
物质谱技术在修饰性酶底物䇥选及动力学常数测定中的应用, 主要内容包括基于蛋白库和肽段库的体外底物篎
选、细胞体内底物䇻选以及动力学常数通量化测定 4 个方面. 此外, 还对生物质谱在该领域中的发展进行了展望.

关键词质谱, 定量蛋白质组学, 翻译后修饰, 修饰性酶, 酶促动力学, 酶促反应

翻译后修饰, 是指蛋白质被翻译以后的化学修饰, 包括磷酸化、乙酰化、糖基化和甲基化等侧链上的 化学修饰以及蛋白质主链的选择性酶切 ${ }^{[1 \sim 3]}$. 已知的 蛋白质翻译后修饰已经超过 300 种, 通过修饰性酶的 双向调控作用下发生动态可逆变化, 从而在调节蛋白 质的活性、结构和功能上发挥着非常重要的作用. 例 如, 其中非常重要的一种蛋白质翻译后修饰——磷酸 化修饰, 底物蛋白在蛋白激酶和磷酸酯酶的双向调控 中发生动态可逆的磷酸化与去磷酸化 ${ }^{[4]}$, 蛋白质由于 被磷酸化而改变其三级结构, 从而改变蛋白质的许多 性质如酶活性、与其他蛋白质的相互作用、在细胞 的亚定位、泛素化等, 进而调控细胞的功能 ${ }^{[5,6]}$. 使蛋 白质发生磷酸化的酶是蛋白激酶, 激酶磷酸化底物蛋 白质, 底物蛋白质也可能是激酶, 可以在激活的条件下
进一步激活下游蛋白质, 这些酶与底物蛋白质之间的 特异性磷酸化相互作用构成了复杂的磷酸化调控网 络, 异常的蛋白质磷酸化表达及变化与许多疾病的发 生有着千丝万缕的关系 ${ }^{[7,8]}$. 人类的疾病, 从根本上来 说大多数都与体内修饰性酶活性异常有关, 理解修饰 酶相关信号调控网络的一个重要基础是揭示酶与底 物之间的对应关系, 其研究成果可以为抑制剂篮选、 药物开发和临床医学提供许多有效信息 ${ }^{[7,9 \sim 12]}$. 例如, Shishkova等人 ${ }^{[13]}$ 通过定量蛋白质组学技术对甲基化 转移酶CARM1 底物进行系统篮选后, 鉴定到 300 个以 上体内CARM 1 相关的精氨酸甲基化位点, 并且 $90 \%$ 以 上底物在体外得到验证. 在这基础上, 该研究组发现 除了 CARM1催化结构域之外, CARM1蛋白 $\mathrm{N}$ 端与底 物的识别和底物发生甲基化修饰的过程也密切相关,

引用格式: 邓真真, 王垄, 叶明亮. 生物质谱在修饰酶底物笁选及动力学常数测定中的应用. 中国科学: 生命科学, 2018, 48: 151-159 Deng Z Z, Wang Y, Ye M L. Mass spectrometry based methods for the enzyme substrate screening and kinetics analysis (in Chinese). Sci Sin Vitae, 2018, 48: 151-159, doi: 10.1360/N052017-00161 
从而为CARM1 抑制剂的开发提供新思路. 因此, 发展 通量化针对修饰性酶在复杂体系中的动力学研究方 法, 例如, 底物的篮选以及底物催化效率常数的测定 方法等, 将有助于提高蛋白质翻译后修饰研究水平.

在研究酶促反应体系时, 最关键的是监测酶底物 的减少与消失或者产物的形成与增加, 因此从大类上 可以分为定性研究与定量研究. 定性研究, 属于发现 式的研究方法, 主要通过与初始样品状态的对比, 发 现消失的反应物或者新生成的产物来对酶促体系进 行研究, 多应用于简单的单底物单个酶的酶促体系. 定量研究则相对比较灵活, 从定量方式上可以分为绝 对定量和相对定量. 但由于绝对定量的方法需要已知 浓度的底物或者产物作为内标, 而对于复杂反应体系, 制备所有底物或其对应产物的绝对内标成本高且费 时费力, 因此多数情况下主要通过对比两个酶促反应 时间点之间的反应物或者产物的浓度变化, 利用相对 定量信息去分析酶底物特异性信息、酶活性以及酶 底物的动力学信息. 其中, 底物催化效率常数的大小 与复杂体系中酶作用在不同底物的相对反应速率呈 正相关, 准确地测量相应的催化效率常数可以为酶底 物篎选提供量化的理论依据, 实现精准化修饰性酶底 物及抑制剂篮选. 生物质谱技术由于其高通量、高灵 敏度、高分辨率的优势, 可以轻松实现对高低丰度或 翻译后修饰等各种底物的监测, 在修饰性酶动力学研 究中发挥着不可忽视的作用 ${ }^{[1,14,15]}$. 本文简要综述了近 几年生物质谱技术在蛋白质组学修饰性酶底物筛选 及动力学研究中的应用, 并对其发展前景进行展望.

\section{1 以蛋白库为篮选库的底物篮选方法}

修饰酶底物䇥选的生物学方法的缺点包括实验 周期长、劳动强度大, 无法实现通量化篮选、鉴定等. 尽管体外酶促实验方法不能完全反映体内的过程, 所 确定的底物只能说是潜在底物, 但是凭其简单、快速 的优点, 在通量化篎选、鉴定修饰性酶底物中获得了 广泛的应用. 现代蛋白质组学技术用于篮选修饰性酶 底物的方法, 能够实现修饰性酶底物和被修饰位点的 同时鉴定, 这为修饰性酶的底物節选提供有效途径.

以磷酸化修饰为例, 一个蛋白质可以被多个激酶 磷酸化, 因此是多个激酶的底物, 但是它们的作用位 点即磷酸化位点往往是不同的. 判断一个蛋白质是否
为某一激酶潜在底物的最常用、最简单的方法是体 外磷酸化. 在这种方法中, 将一种激酶与细胞的总蛋 白质提取液和三磷酸腺苷(adenosine triphosphate, ATP) 共同孵育一段时间, 经过分析鉴定, 那些被磷酸化的 蛋白质就是潜在的底物蛋白质. 但是这种方法存在一 个很大缺陷, 由于细胞中的总提取物中本来就包含很 多激酶, 当加入ATP孵育后这些激酶也将使它们的底 物蛋白质发生磷酸化, 从而被目标激酶磷酸化的蛋白 质不能被特异性监测、识别出来. 为了解决由于这种 内在的激酶造成的背景磷酸化的干扰, 目前已经发展 了很多方法,包括基因工程改造激酶 ${ }^{[16-18]}$, 激酶底物追 踪与解析 ${ }^{[19]}$, 蛋白质组芯片 ${ }^{[20]}$ 等. Zhang等人 ${ }^{[21]}$ 将细胞 提取物中的总蛋白质固载于固相微球上进行体外磷 酸化, 细胞提取液中的干扰激酶在被固载后, 与其他 蛋白质相互作用的机会大幅度降低而难以使其他蛋 白质发生磷酸化, 从而有效抑制背景磷酸化对激酶底 物篎选的影响. 进一步利用磷酸化蛋白质组学分析技 术鉴定潜在的激酶底物蛋白及其磷酸化位点, 实现通 量化鉴定激酶底物蛋白质及其作用磷酸化位点的目 的. 与其他同类方法相比, 该方法更简单经济, 并且该 固相方法同样适用于其他修饰性酶底物的研究. Bian 等人 ${ }^{[22]}$ 在Zhang 等人 ${ }^{[21]}$ 方法基础上, 将体外固相激酶 反应、定量蛋白质组学和规模化磷酸化位点数据库 的应用相结合, 发展了一个整合的磷酸化蛋白质组学 分析流程, 用于 CK2 激酶底物蛋白的高通量规模化篎 选, 从 356 个蛋白质中鉴定到了 605 个高可信度的潜在 底物位点.

除了蛋白质磷酸化以外, 生物体内还有蛋白质乙 酰化、甲基化、糖基化等三百多种翻译后修饰, 确定 其他蛋白质修饰酶与底物之间的关系也非常重要. 蛋 白质的选择性酶切是一种特殊的蛋白质翻译后修饰, 它可以通过特异性水解蛋白质从而不可逆地改变蛋 白质的性质和功能, 调节细胞的生长、代谢、死亡及 免疫等过程 ${ }^{[23]}$. 人类蛋白酶数量超过 500 个, 大约占人 类基因组的 $2 \%$, 许多遗传病被发现与蛋白质的特异性 水解有关 ${ }^{[24]}$. 例如, 含半胱氨酸的天冬氨酸蛋白水解 酶(caspase), 在细胞调亡、分化、炎症反应等过程中 发挥着重要作用 ${ }^{[25,26]}$, 因此对其研究备受生物化学家 和医学家青睐. 大规模笁选 caspases底物蛋白已经取 得一定进展 ${ }^{[26-30]}$, 这些数据对于癌症治疗、神经退行 性疾病等方面的研究具有重要的指导意义. 因此, 发 
展高通量蛋白酶底物分析方法, 了解蛋白酶与其底物 之间的关系, 可以提供整个蛋白降解网络信息, 有利于 实现蛋白酶靶向的相关疾病治疗 ${ }^{[31]}$. 与磷酸化、糖基 化等这类可以通过亲和富集方法进行直接提取的修 饰性肽段不同, 由蛋白酶水解产生的肽段存在于大量 背景蛋白质中, 如何特异性地提取相应酶切肽段, 排 除鉴定时的背景干扰是实现蛋白酶底物篎选的关键. 基于凝胶电泳的方法 ${ }^{[32]}$, 是将蛋白酶处理与不处理的 样品蛋白进行分离从而找出差异蛋白点, 进而采用质 谱鉴定或者定量蛋白质组学方法篮选底物蛋白, 该类 方法没有对底物蛋白或者酶切肽段进行特异性富集, 在大量背景干扰之下, 鉴定到的蛋白酶底物对应的蛋 白丰度通常相对较高. Wang 等人 ${ }^{[33]}$ 发展了一种基于 固相酶切反应的蛋白酶底物的体外篮选方法(图1). 该 方法直接将蛋白裂解液中的蛋白质固载在固相载体 上作为底物篎选库, 然后加入蛋白酶进行反应, 底物 蛋白被特异性酶切之后生成产物肽段进入溶液中, 同 时非底物蛋白仍被完整地保留在固相载体上, 通过离 心分离就可以得到由目标蛋白酶酶解产生的酶切肽 段. 经过胰蛋白酶二次酶解后, 利用质谱对肽段进行 鉴定. 该研究组将此方法用于调亡执行酶caspase-3的 底物篮选, 采用定量蛋白质组学技术, 最终从急性 $\mathrm{T}$ 细 胞白血病细胞(Jurkat)中篮选出 1098 个体外caspase-3底 物蛋白以及 503 个酶切位点, 对于研究caspase- 3 酶介导 的调亡机理具有重要意义.

Mahrus等人 ${ }^{[28]}$ 发展了一种酶化学方法修饰蛋白 酶处理的 $\mathrm{N}$ 端肽段并进行选择性分离的方法. 该方法 利用 substiligase和含有TEV酶切位点的生物素标记的 肽段与蛋白酶产生的肽段的 $\mathrm{N}$ 端 $\alpha$-伯胺基团进行反应, 经过二次胰蛋白酶酶解后, 采用固载亲和素微球特异 性富集含有修饰的肽段, 再进一步通过TEV酶酶切释
放产物肽段后进行质谱鉴定. 该研究组将此方法成功 应用于依托泊苷(etoposide)诱导的急性 $\mathrm{T}$ 细胞白血病 细胞(Jurkat)调亡过程中caspase底物蛋白的研究, 并成 功䇥选出 292 个 caspase底物蛋白以及 333 个酶切位点. 通过将该方法与选择性反应监测技术(selected reaction monitoring, SRM)相结合, 该研究组还分析了不同药物 诱导不同细胞系调亡过程中 caspase的底物及酶切位 点 ${ }^{[34,35]}$, 通过比较结果发现 caspase酶切位点既具有保 守性, 又具有细胞依赖性或药物特异性.

\section{2 以肽库为篮选库的底物篮选方法}

修饰性酶在催化蛋白发生相应修饰时, 具有高度 特异性的特点. 修饰性酶催化结构域的空间结构与氨 基酸分布往往是影响酶促底物特异性的关键因素, 修 饰性酶催化结构域的空间构象决定了只有具有特定 结构的底物序列才可以被发生催化作用,而也正是酶 催化结构域的氨基酸分布决定了底物序列的亲疏水 性和带电性质等. 因此, 直接研究修饰性酶底物蛋白 的特异性氨基酸序列(motif)对于预测酶底物、揭示修 饰性酶在生物体内发挥的作用同样具有指导意义.

目前在肽段层次上进行修饰性酶底物篎选的方法 很多 ${ }^{[36-38]}$. 基于合成肽库进行特异性分析的方法具有 高通量、高效率的优点 ${ }^{[39]}$, 将合成肽库与质谱结合的 方式可用于分离富集难度较大的修饰性底物的研究, 如蛋白质精氨酸甲基转移酶的底物篎选 ${ }^{[40]}$. 但是人工 合成肽库费时、成本高, 并且肽段序列可能不包含在 现有的数据库中而造成数据分析困难 ${ }^{[41]}$. 采用蛋白质 组衍生化肽段库不仅能够匹配生物体内表达的蛋白, 而且相对于合成肽库构建比较容易, 已被广泛用于修 饰性酶的研究 ${ }^{[42,43]}$. Wang等人 ${ }^{[44]}$ 以经过去磷酸化处理

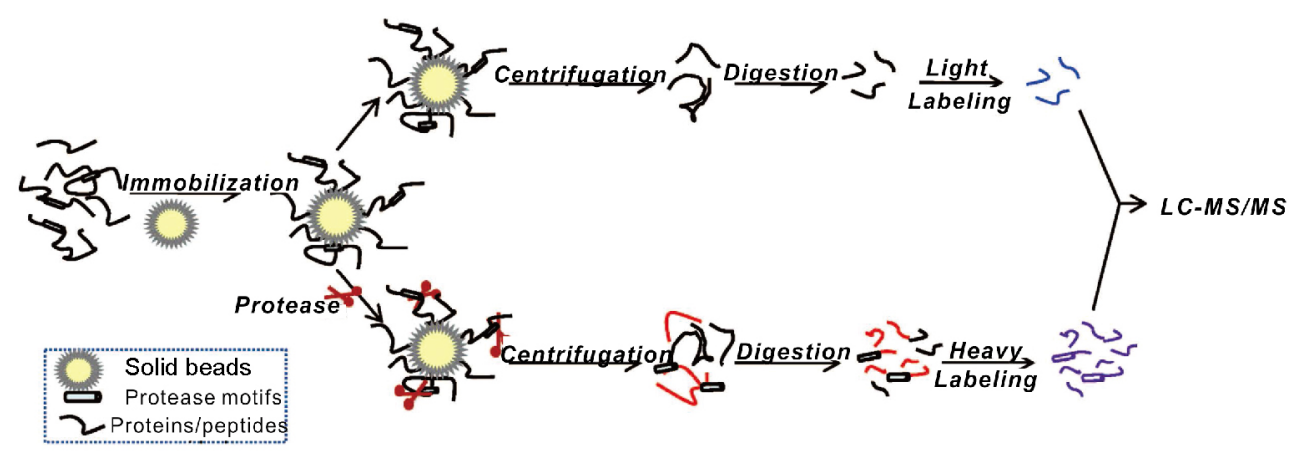

图 1 基于固相酶切反应的蛋白酶底物的篮选方法流程图(网络版彩图) ${ }^{[33]}$ 
的细胞裂解液的胰蛋白酶酶解液作为酶促反应体系, 对酪蛋白激酶2 (casein kinase 2, CK2)激酶进行特异性 研究(图2). 为了除去肽库中内源性磷酸化位点对激酶 特异性研究结果的干扰, 将蛋白质衍生化肽库篎选与 二甲基标记定量技术结合, 将经过激酶处理的样品与 不经过激酶处理的对照组分别进行稳定同位素二甲 基标记, 混合后经过固定钛离子亲和色谱 $\left(\mathrm{Ti}^{4+}-\mathrm{IMAC}\right)$ 材料进行磷酸肽特异性富集后再利用液相色谱质谱 联用系统对体系进行定量与定性分析, 最终成功鉴定 到 404 个磷酸化位点, 可用于 CK2 激酶的特异性研究, 经过分析后最终得到 CK2 激酶的特异性作用模体为 $\mathrm{S} / \mathrm{T}-\mathrm{D} / \mathrm{E}-\mathrm{X}-\mathrm{E} / \mathrm{D}$. 值得注意的是, 即使发现某个蛋白含 有某种激酶的特异性作用底物序列, 并不能说明就是 该对应激酶的底物蛋白, 还需要考虑该蛋白质与激酶 的细胞定位, 以及是否能与激酶发生相互作用, 最终
篮选出高可信度的激酶蛋白底物. 该研究组通过将体 外笁选出的 CK2 底物蛋白与体内磷酸化蛋白质组数据 进行对比, 进行潜在 CK2 激酶底物蛋白篮选, 从而最终 篎选得到105个高可信度的潜在底物蛋白.

采用蛋白质组学衍生肽库进行蛋白酶底物篎选 时, 为了在肽段层次将蛋白酶产生的肽段与背景肽段 进行有效区分, 在降低样片复杂度的同时降低质谱分 析难度, 目前主要发展了一系列基于化学方法修饰的 $N$ 端选择性标记与分离的方法 ${ }^{[45 \sim 48]}$. Schilling和Overall ${ }^{[42]}$ 发展了一种基于蛋白质衍生化肽段库的端基标记方法 PICS (proteomic identification of protease cleavage sites) 用于蛋白酶特异性研究. 基本原理是先将蛋白质酶解 产生的肽段中所有伯胺基团封闭, 作为肽段库与蛋白 酶进行孵育, 反应后利用生物素(biotin)将新产生的 $\mathrm{N}$ 端 酶切肽段进行标记, 进而采用链霉亲和素(streptavidin)

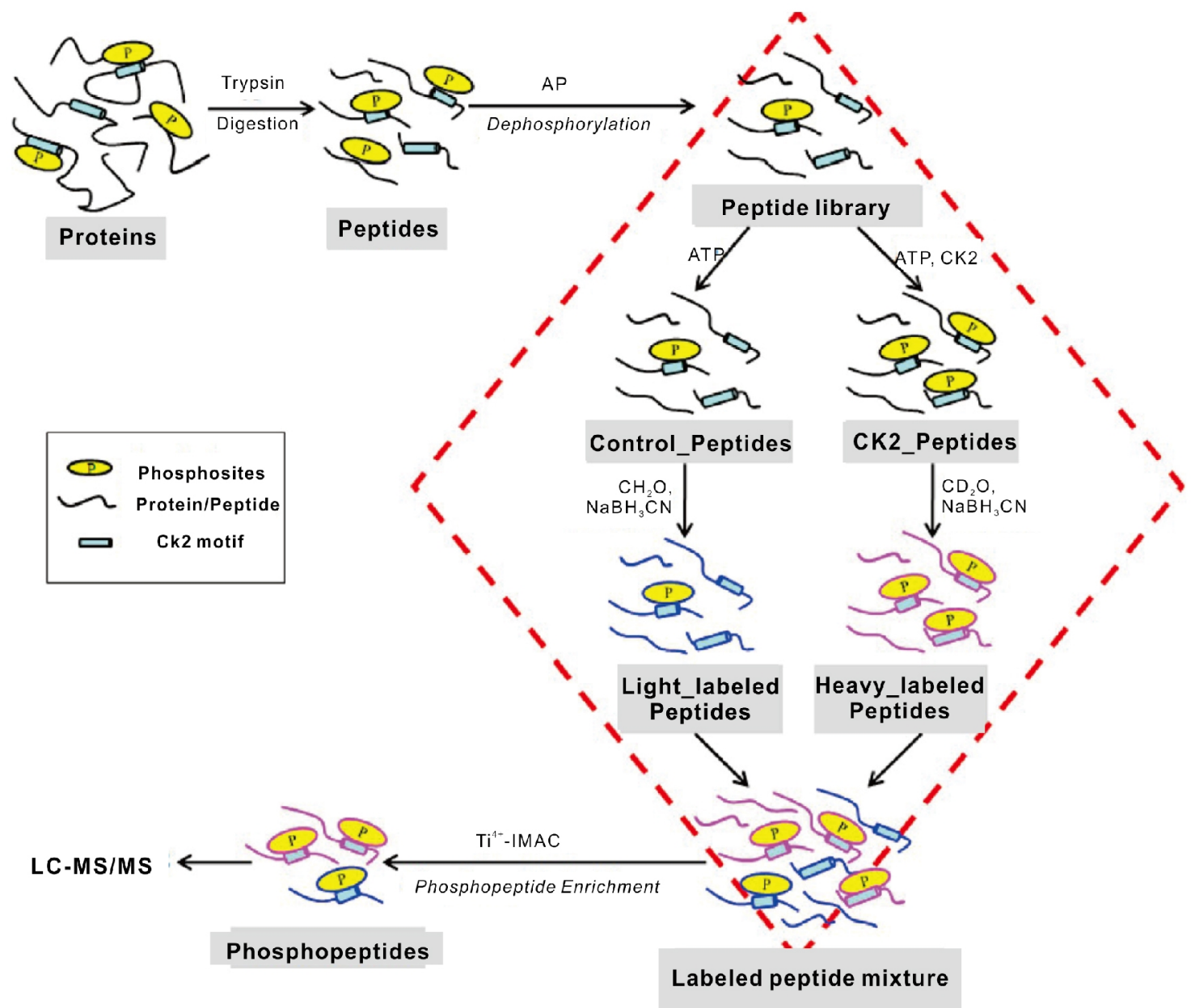

图 2 细胞裂解液构建肽库用于篮选 CK2 激酶特异性的工作流程图 (网络版彩图) ) $^{[44]}$ 
微球将生物素标记的肽段进行特异性富集, 最后利用 质谱对洗脱肽段进行分析. 该方法与传统合成肽库相 比, 不仅避免复杂的操作过程, 还能获得准确的蛋白 酶切位点信息, 而且由于蛋白质衍生化肽段库来源于 生物体本身表达的蛋白, 该方法确定的蛋白酶特异性 信息对于篮选底物提供的信息更有价值.

\section{3 细胞体内底物篮选方法}

基于质谱的修饰性酶底物蛋白细胞体内篮选技 术, 主要是通过建立修饰性酶过表达、酶活性抑制或 者酶敲除等体系, 进一步结合定量蛋白质组学方法来 实现 ${ }^{[13,49 \sim 51]}$. Franz-Wachtel等人 ${ }^{[49]}$ 构建了一个蛋白激 酶PKD1持续保持活性以及没有活性的两株细胞系体 系, 用于体内 PKD1底物蛋白的節选(图3). 将PKD1的 活化剂处理以上两株SILAC标记的细胞, 等量混合后 进行蛋白提取及酶解, 利用磷酸化蛋白质组学技术进 行后续磷酸肽定量与定性分析, 最终在没有PKD1激 酶活性的细胞中鉴定到124个丰度明显下调的磷酸化 位点. 将这些鉴定到的位点氨基酸序列与PKD1激酶 已知的底物序列进行对比, 发现具有很高的相似度, 证明该方法鉴定到的底物蛋白具有较高的可信度. 但 是由于细胞中蛋白激酶调控网络非常复杂, 通过多级 串联和多通路交叉反应, 大部分蛋白激酶都受到细胞 信号传导通路调控, 因此采用体内䇻选方法得到的蛋 白质在理论上只能属于受该目标激酶调控的蛋白质, 要证明这个底物蛋白属于该激酶的直接底物, 还需要 获得更多数据支持. Shishkova等人 ${ }^{[13]}$ 通过构建甲基化 转移酶CARM1保持活性以及没有活性的细胞系体系, 结合基于串联质量标签(tandem mass tag, TMT)的定量 质谱技术用于CARM1酶底物特异性研究. 为了提高 质谱分析灵敏度, 该研究组采用不对称二甲基精氨酸 抗体对 CARM1酶底物进行特异性富集, 最终鉴定到 300 个以上体内 CARM 1 相关的精氨酸甲基化位点以及 130 个以上的底物蛋白.

\section{4 酶促动力学常数的通量化测定方法}

底物催化效率常数 $\left(k_{\mathrm{cat}} / K_{\mathrm{m}}\right)$ 的大小与复杂体系中 酶作用在不同底物的相对反应速率呈正相关, 因而可 为酶底物篎选提供量化的理论依据 ${ }^{[52]}$, 对酶促反应体 系中的底物进行催化效率常数的计算可以实现酶底

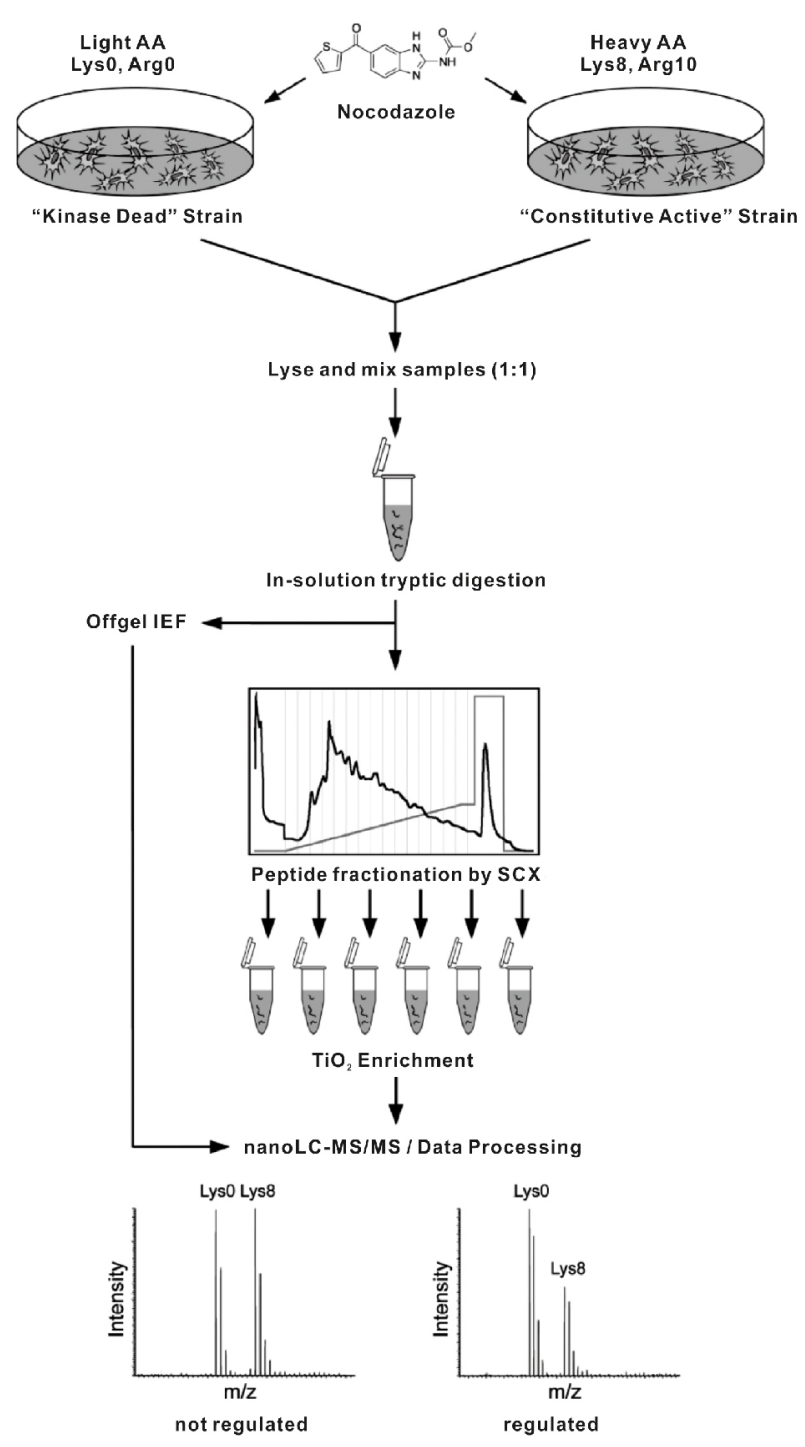

图 3 基于激酶活差异细胞株用于体内激酶底物蛋白 篮选的流程图 ${ }^{[49]}$

物的精准篮选, 为酶抑制剂和相应药物的设计研发提 供更详细的信息 ${ }^{[34,53,54]}$.

Agard 等人 ${ }^{[34]}$ 将 $\mathrm{N}$ 端肽段富集技术 ${ }^{[5]}$ 与 $\mathrm{SRM}$ 技术 ${ }^{[56]}$ 相结合, 让质谱仅对目标肽段进行质谱信号 采集, 从而获取准确定量信息, 并将该方法应用于 caspase蛋白酶的动力学研究, 对于反应前后丰度变化 相差 500 倍以上的底物也能实现准确定量. 该方法能 监测上百种底物随反应时间的相对丰度变化, 将数据 代入准一级动力学方程后可以得到每个底物对应的 催化效率常数. 唯一不足的是, 该研究组并没有详细 说明在复杂体系底物催化效率常数计算中准一级动 
力学方程的具体使用条件.

经典米氏方程及其衍生化方程是进行评价酶与 底物催化效率大小及酶选择性的基础 ${ }^{[57]}$, 但都是基于 一个纯化的底物与一个酶之间的酶促反应，如果要将 衍生化方程用于复杂体系, 例如, 单个酶与多个底物 反应体系时, 需要重新对动力学方程进行推导, 从而 获得正确的对应关系及方程使用条件. Deng等人 ${ }^{[54]}$ 以米氏方程为基础进行衍生化处理，构建复杂体系酶 促反应方程组(图4), 发现在任意一个反应时间点下, 各个底物通过其剩余游离底物浓度对游离酶浓度的 影响, 进而对其余底物的酶促反应速率造成影响, 而 各个底物的酶促反应速率又反过来与下一个时间点 各个底物的剩余浓度相关, 以此循环阻止方程的进一 步简化推导. 为了解决这个问题, 该研究组对复杂体 系中的酶促反应进行微分处理, 将各个因素之间的相 互制约顺序化, 从而建立复杂酶促体系酶促动力学 理论模型. 发现当满足 $\sum \frac{\left[S_{x}\right]}{K_{M}^{X}} \ll 1$ (体系中所有底物 浓度与其相应米氏常数比值的加和远小于 1)条件时, 可以用准一级动力学方程描述每个底物的反应, 从 而实现底物催化效率的通量化测定. 通过对不同酶 反应时间的样品进行二甲基标记以及质谱分析，得到
每个底物经过不同酶促反应时间对应的相对丰度变 化, 最终成功鉴定到 2369 个蛋白酶底物并计算出相应 催化效率常数.

\section{5 展望}

现有的与生物质谱相结合用于修饰酶底物篮选 方法种类繁多, 本研究组已经得到大量关于各种修饰 性酶的底物信息库, 由于大部分底物特异性信息是基 于体外笁选方法获得, 在真实环境中由于位置等因素 并不一定能与相应修饰性酶发生相互作用, 假阳性率 较高. 确定底物蛋白位点的生物学功能, 具有非常重 要的生物学意义, 在越来越庞大的底物数据库中如何 进行快速选择, 针对少量位点进行深入生物学研究就 变得非常关键. 通过与规模化体内实验得到的位点数 据库比对确实可以提高位点可信度, 另一方面, 结合 定量蛋白质组学技术进行修饰性酶底物精准化的动 力学篮选, 根据催化效率常数评价各个底物蛋白与酶 之间相互作用特异性强弱, 也可以为底物蛋白笁选提 供强有力的依据. 因此, 建立简单、准确、高通量的 适用于复杂体系酶底物笁选的动力学方法, 可以推进 修饰性酶生物功能底物研究进展.

$$
\left\{\begin{array}{l}
\mathrm{E}+\mathrm{S}_{\mathrm{a}} \frac{k_{1}^{a}}{k_{-1}^{a}} \mathrm{ES}_{\mathrm{A}} \frac{k_{c a t}^{a}}{\mathrm{E}}+\mathrm{P}_{\mathrm{a}} \\
\mathrm{E}+\mathrm{S}_{\mathrm{b}} \frac{k_{1}^{b}}{k_{-1}^{b}} \mathrm{ES}_{\mathrm{b}} \frac{k_{c a t}^{b}}{\mathrm{E}}+\mathrm{P}_{\mathrm{b}} \\
\mathrm{E}+\mathrm{S}_{\mathrm{c}} \frac{k_{1}^{c}}{k_{-1}^{c}} \mathrm{ES}_{\mathrm{c}} \frac{k_{c a t}^{c}}{\mathrm{E}}+\mathrm{P}_{\mathrm{c}} \\
\mathrm{E}+\mathrm{S}_{\mathrm{d}} \frac{k_{1}^{d}}{k_{-1}^{d}} \mathrm{ES}_{\mathrm{d}} \frac{k_{c a t}^{d}}{\cdots}+\mathrm{P}_{\mathrm{d}} \\
\mathrm{E}+\mathrm{S}_{\mathrm{i}} \frac{k_{1}^{i}}{k_{-1}^{i}} \mathrm{ES}_{\mathrm{i}} \frac{k_{c a t}^{i}}{\ldots}+\mathrm{P}_{\mathrm{i}}
\end{array}\right.
$$

$$
\begin{gathered}
{[\mathrm{E}]_{\text {free }}=\frac{1}{\left(1+\sum_{\mathrm{x}=1}^{\mathrm{n}} \frac{\left[\mathrm{S}_{\mathrm{x}}\right]}{K_{m}^{x}}\right)}} \\
v_{i}=\frac{\mathrm{d}\left[\mathrm{S}_{\mathrm{i}}\right]}{\mathrm{d} t}=-\frac{\mathrm{d}\left[\mathrm{P}_{\mathrm{i}}\right]}{\mathrm{d} t}=k_{\text {cat }}^{i} \frac{[\mathrm{E}]_{\text {free }}\left[\mathrm{S}_{\mathrm{i}}\right]}{K_{m}^{i}} \\
=-\frac{k_{c a t}^{i}}{K_{m}^{i}\left(1+\sum_{\mathrm{x}=1}^{\mathrm{n}} \frac{\left[\mathrm{S}_{\mathrm{x}}\right]}{K_{m}^{x}}\right)}[\mathrm{E}]_{\mathrm{tot}}\left[\mathrm{S}_{\mathrm{i}}\right] \\
\square \text { If } \sum_{\mathrm{x}=1}^{\mathrm{n}} \frac{\left[\mathrm{S}_{\mathrm{x}}\right]}{K_{m}^{x}} \ll 1
\end{gathered}
$$

Enzymatic reactions in complex system

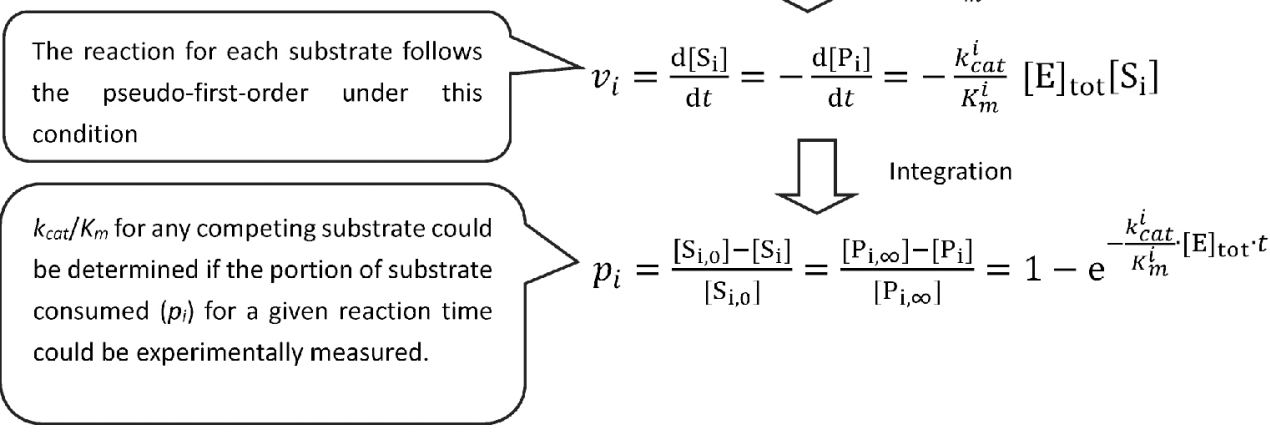

图 4 用于复杂体系底物催化效率的计算的酶促动力学模型示意图 (网络版彩图) ${ }^{[54]}$ 


\section{参考文献}

1 Wilhelm M, Schlegl J, Hahne H, et al. Mass-spectrometry-based draft of the human proteome. Nature, 2014, 509: 582-587

2 Aebersold R, Mann M. Mass spectrometry-based proteomics. Nature, 2003, 422: 198-207

3 Pandey A, Mann M. Proteomics to study genes and genomes. Nature, 2000, 405: 837-846

4 Westphal R S. Regulation of NMDA receptors by an associated phosphatase-kinase signaling complex. Science, 1999, 285: 93-96

5 Blume-Jensen P, Hunter T. Oncogenic kinase signalling. Nature, 2001, 411: 355-365

6 Lim W A, Pawson T. Phosphotyrosine signaling: evolving a new cellular communication system. Cell, 2010, 142: 661-667

7 Shukla D, Meng Y, Roux B, et al. Activation pathway of Src kinase reveals intermediate states as targets for drug design. Nat Commun, 2014, 5: $3397-3407$

8 Webb B A, Forouhar F, Szu F E, et al. Structures of human phosphofructokinase-1 and atomic basis of cancer-associated mutations. Nature, 2015, 523: 111-114

9 Patterson A W, Wood W J L, Ellman J A. Substrate activity screening (SAS): a general procedure for the preparation and screening of a fragmentbased non-peptidic protease substrate library for inhibitor discovery. Nat Protoc, 2007, 2: 424-433

10 Zhang J, Yang P L, Gray N S. Targeting cancer with small molecule kinase inhibitors. Nat Rev Cancer, 2009, 9: 28-39

11 Kho C, Lee A, Jeong D, et al. Small-molecule activation of SERCA2a SUMOylation for the treatment of heart failure. Nat Commun, 2015, 6: 7229-7239

12 Yang L, Xie M, Yang M, et al. Pkm2 regulates the warburg effect and promotes hmgb1 release in sepsis. Nat Commun, 2014, 5: 4436-4444

13 Shishkova E, Zeng H, Liu F, et al. Global mapping of carm1 substrates defines enzyme specificity and substrate recognition. Nat Commun, 2017, 8: $15571-15583$

14 Domon B, Aebersold R. Mass spectrometry and protein analysis. Science, 2006, 312: 212-217

15 Schubert O T, Röst H L, Collins B C, et al. Quantitative proteomics: challenges and opportunities in basic and applied research. Nat Protoc, 2017, 12: 1289-1294

16 Cohen P, Knebel A. KESTREL: a powerful method for identifying the physiological substrates of protein kinases. Biochem J, 2006, 393: 1-6

17 Kumar N V, Eblen S T, Weber M J. Identifying specific kinase substrates through engineered kinases and ATP analogs. Methods, 2004, 32: 389-397

18 Sivars U, Aivazian D, Pfeffer S R. Yip3 catalyses the dissociation of endosomal Rab-GDI complexes. Nature, 2003, 425: 856-859

19 Huang S Y, Tsai M L, Chen G Y, et al. A systematic MS-based approach for identifying in vitro substrates of PKA and PKG in rat uteri. J Proteome Res, 2007, 6: 2674-2684

20 Ptacek J, Devgan G, Michaud G, et al. Global analysis of protein phosphorylation in yeast. Nature, 2005, 438: 679-684

21 Zhang M, Han G, Wang C, et al. A bead-based approach for large-scale identification of in vitro kinase substrates. Proteomics, 2011, 11: 4632-4637

22 Bian Y, Ye M, Wang C, et al. Global screening of CK2 kinase substrates by an integrated phosphoproteomics workflow. Sci Rep, 2013, 3: 1611-1614

23 Barrett A J. Bioinformatics of proteases in the merops database. Curr Opin Drug Disco Devel, 2004, 7: 334-341

24 Puente X S, Sánchez L M, Overall C M, et al. Human and mouse proteases: a comparative genomic approach. Nat Rev Genet, 2003, 4: 544-558

25 Lamkanfi M, Festjens N, Declercq W, et al. Caspases in cell survival, proliferation and differentiation. Cell Death Differ, 2007, 14: 44-55

26 Lüthi A U, Martin S J. The CASBAH: a searchable database of caspase substrates. Cell Death Differ, 2007, 14: 641-650

27 Dix M M, Simon G M, Cravatt B F. Global mapping of the topography and magnitude of proteolytic events in apoptosis. Cell, 2008, 134: 679-691

28 Mahrus S, Trinidad J C, Barkan D T, et al. Global sequencing of proteolytic cleavage sites in apoptosis by specific labeling of protein N termini. Cell, 2008, 134: 866-876

29 Timmer J C, Salvesen G S. Caspase substrates. Cell Death Differ, 2007, 14: 66-72

30 Julien O, Zhuang M, Wiita A P, et al. Quantitative MS-based enzymology of caspases reveals distinct protein substrate specificities, hierarchies, and cellular roles. Proc Natl Acad Sci USA, 2016, 113: E2001-E2010

31 Doucet A, Butler G S, Rodríguez D, et al. Metadegradomics. Mol Cell Proteomics, 2008, 7: 1925-1951

32 Lee A Y, Park B C, Jang M, et al. Identification of caspase-3 degradome by two-dimensional gel electrophoresis and matrix-assisted laser desorption/ionization-time of flight analysis. Proteomics, 2004, 4: 3429-3436

33 Wang C, Ye M, Wei X, et al. A bead-based cleavage method for large-scale identification of protease substrates. Sci Rep, 2016, 6: 22645-22653

34 Agard N J, Mahrus S, Trinidad J C, et al. Global kinetic analysis of proteolysis via quantitative targeted proteomics. Proc Natl Acad Sci USA, 2012, 109: 1913-1918 
35 Shimbo K, Hsu G W, Nguyen H, et al. Quantitative profiling of caspase-cleaved substrates reveals different drug-induced and cell-type patterns in apoptosis. Proc Natl Acad Sci USA, 2012, 109: 12432-12437

36 Songyang Z, Blechner S, Hoagland N, et al. Use of an oriented peptide library to determine the optimal substrates of protein kinases. Curr Biol, 1994, 4: 973-982

37 Mok J, Kim P M, Lam H Y K, et al. Deciphering protein kinase specificity through large-scale analysis of yeast phosphorylation site motifs. Sci Signal, 2010, 3: ra12

38 Hutti J E, Jarrell E T, Chang J D, et al. A rapid method for determining protein kinase phosphorylation specificity. Nat Meth, 2004, 1: 27-29

39 Yu Y, Anjum R, Kubota K, et al. A site-specific, multiplexed kinase activity assay using stable-isotope dilution and high-resolution mass spectrometry. Proc Natl Acad Sci USA, 2009, 106: 11606-11611

40 Osborne T C, Obianyo O, Zhang X, et al. Protein arginine methyltransferase 1: positively charged residues in substrate peptides distal to the site of methylation are important for substrate binding and catalysis. Biochemistry, 2007, 46: 13370-13381

41 Perkins D N, Pappin D J C, Creasy D M, et al. Probability-based protein identification by searching sequence databases using mass spectrometry data. Electrophoresis, 1999, 20: 3551-3567

42 Schilling O, Overall C M. Proteome-derived, database-searchable peptide libraries for identifying protease cleavage sites. Nat Biotechnol, 2008, 26: $685-694$

43 Xue L, Wang W, Lliuk A, et al. Sensitive kinase assay linked with phosphoproteomics for identifying direct kinase substrates. Proc Natl Acad Sci USA, 2012, 109: 5616-5620

44 Wang C, Ye M, Bian Y, et al. Determination of CK2 specificity and substrates by proteome-derived peptide libraries. J Proteome Res, 2013, 12: 3813-3821

45 Gevaert K, Goethals M, Martens L, et al. Exploring proteomes and analyzing protein processing by mass spectrometric identification of sorted n-terminal peptides. Nat Biotechnol, 2003, 21: 566-569

46 Staes A, Van Damme P, Helsens K, et al. Improved recovery of proteome-informative, protein N-terminal peptides by combined fractional diagonal chromatography (COFRADIC). Proteomics, 2008, 8: 1362-1370

47 Van Damme P, Martens L, Van Damme J, et al. Caspase-specific and nonspecific in vivo protein processing during Fas-induced apoptosis. Nat Meth, 2005, 2: 771-777

48 McDonald L, Beynon R J. Positional proteomics: preparation of amino-terminal peptides as a strategy for proteome simplification and characterization. Nat Protoc, 2006, 1: 1790-1798

49 Franz-Wachtel M, Eisler S A, Krug K, et al. Global detection of protein kinase D-dependent phosphorylation events in nocodazole-treated human cells. Mol Cell Proteomics, 2012, 11: 160-170

50 Wang P, Xue L, Batelli G, et al. Quantitative phosphoproteomics identifies SnRK2 protein kinase substrates and reveals the effectors of abscisic acid action. Proc Natl Acad Sci USA, 2013, 110: 11205-11210

51 Imamura $\mathrm{H}$, Wagih $\mathrm{O}$, Niinae $\mathrm{T}$, et al. Identifications of putative PKA substrates with quantitative phosphoproteomics and primary-sequencebased scoring. J Proteome Res, 2017, 16: 1825-1830

52 Eisenthal R, Danson M J, Hough D W. Catalytic efficiency and $k_{\text {cat }} / k_{\mathrm{m}}$ : a useful comparator? Trends Biotechnol, 2007, 25: 247-249

53 Meyer N O, O'Donoghue A J, Schulze-Gahmen U, et al. Multiplex substrate profiling by mass spectrometry for kinases as a method for revealing quantitative substrate motifs. Anal Chem, 2017, 89: 4550-4558

54 Deng Z, Mao J, Wang Y, et al. Enzyme kinetics for complex system enables accurate determination of specificity constants of numerous substrates in a mixture by proteomics platform. Mol Cell Proteomics, 2017, 16: 135-145

55 Michaelis L, Menten M L. Die kinetik der invertinwirkung. Biochem, 1913, 49: 333-369

56 Agard N J, Maltby D, Wells J A. Inflammatory stimuli regulate caspase substrate profiles. Mol Cell Proteomics 2010, 9: 880-893

57 Picotti P, Aebersold R. Selected reaction monitoring-based proteomics: workflows, potential, pitfalls and future directions. Nat Meth, 2012, 9: $555-566$ 


\title{
Mass spectrometry based methods for the enzyme substrate screening and kinetics analysis
}

\author{
DENG ZhenZhen ${ }^{1,2}$, WANG Yan ${ }^{1,2}$ \& YE MingLiang ${ }^{1}$ \\ ${ }^{1}$ National Chromatography R\&A Center, Key Laboratory of Separation Science for Analytical \\ Chemistry, Dalian Institute of Chemical Physics, Chinese Academy of Sciences, Dalian 116023, China; \\ ${ }^{2}$ School of Chemical Science, University of Chinese Academy of Sciences, Beijing 100049, China
}

Revealing the relationship between the enzyme and substrate could provide insights into signaling networks. Mass spectrometry has been widely applied for the large scale interrogation of biological systems in proteomics, and also has taken an important role in determination of enzyme activities and screening of enzyme inhibitors. In this review, we introduced the recent advance in the mass spectrometry based methods for enzyme substrate screening and enzyme kinetics analysis, including in vitro substrate screening using protein-derived library and peptide-derived library, in vivo substrate screening and high-throughput kinetics analysis, and then gave our opinions on the outlook of mass spectrometry in the study of enzyme kinetics in complex system.

mass spectrometry (MS), quantitative proteomics, post-translational modification, modified enzyme, enzymatic kinetics, enzymatic reaction

doi: $10.1360 / \mathrm{N} 052017-00161$ 